Ishii H, Seymour JF, Tazawa R, et al. Secondary pulmonary alveolar proteinosis complicating myelodysplastic syndrome results in worsening of prognosis: a retrospective cohort study in Japan. BMC Pulm Med 2014; $14: 37$.

3 Inoue $\mathrm{Y}$, Trapnell BC, Tazawa R, et al. Characteristics of a large cohort of patients with autoimmune pulmonary alveolar proteinosis in Japan. Am J Respir Crit Care Med 2008; 177: 752-762.

4 Griese M, Zarbock R, Costabel U, et al. GATA2 deficiency in children and adults with severe pulmonary alveolar proteinosis and hematologic disorders. BMC Pulm Med 2015; 15: 87.

5 Marchand-Adam S, Diot B, Magro P, et al. Pulmonary alveolar proteinosis revealing a telomerase disease. Am J Respir Crit Care Med 2013; 188: 402-404.

6 Luisetti M, Bruno P, Kadija Z, et al. Relationship between diffuse pulmonary fibrosis, alveolar proteinosis, and granulocyte-macrophage colony stimulating factor autoantibodies. Respir Care 2011; 56: 1608-1610.

7 Ishii H, Tazawa R, Kaneko C, et al. Clinical features of secondary pulmonary alveolar proteinosis: pre-mortem cases in Japan. Eur Respir J 2011; 37: 465-468.

8 Ishii H, Trapnell BC, Tazawa R, et al. Comparative study of high-resolution CT findings between autoimmune and secondary pulmonary alveolar proteinosis. Chest 2009; 136: 1348-1355.

9 Briens E, Delaval P, Mairesse MP, et al. Lipoproteinose alveolaire pulmonaire. [Pulmonary alveolar proteinosis.] Rev Mal Respir 2002; 19: 166-182.

10 Spinner MA, Sanchez LA, Hsu AP, et al. GATA2 deficiency: a protean disorder of hematopoiesis, lymphatics, and immunity. Blood 2014; 123: 809-821.

11 Cuellar-Rodriguez J, Gea-Banacloche J, Freeman AF, et al. Successful allogeneic hematopoietic stem cell transplantation for GATA2 deficiency. Blood 2011; 118: 3715-3720.

\title{
Bosutinib therapy resulting in severe deterioration of pre-existing pulmonary arterial hypertension
}

To the Editor:

We read with interest the recent correspondence from QuiLOT et al. [1] describing a likely case of ponatinib-associated pulmonary arterial hypertension (PAH). The authors hypothesised that the mechanism by which tyrosine kinase inhibitors (TKIs) such as dasatinib and ponatinib, used for the treatment of chronic myeloid leukaemia (CML), induce PAH may involve their common inhibition of the non-receptor tyrosine kinase, Src [1]. Here, we present a patient who developed marked worsening of pre-existing TKI-associated PAH following commencement of bosutinib, a third-generation TKI also known to inhibit Src [2]. After marked improvement on withdrawal of bosutinib, the patient experienced further significant worsening of PAH after commencing ponatinib. We believe this is the first reported case linking bosutinib with PAH. It also supports the association between ponatinib and $\mathrm{PAH}$ and represents the first time that the development or worsening of PAH associated with multiple TKIs in the same patient has been reported.

A 39-year-old man was diagnosed with CML in 2001. He had no other medical history of note. Following initial disease control with imatinib (March 2004-October 2009), he was switched to dasatinib because of loss of cytogenetic response. After 12 months of therapy, he became progressively breathless and developed pleural effusions. He was given diuretics and dasatinib was stopped in January 2011. Despite resolution of the pleural effusions and initial improvement in symptoms, he subsequently became more breathless and was assessed in our pulmonary hypertension (PH) unit in March 2011. Right heart catheterisation (RHC) confirmed severe $\mathrm{PAH}$ (mean right atrial pressure $15 \mathrm{mmHg}$; mean pulmonary arterial pressure $55 \mathrm{mmHg}$; pulmonary arterial wedge pressure $11 \mathrm{mmHg}$; cardiac index $2.5 \mathrm{~L} \cdot \mathrm{min}^{-1} \cdot \mathrm{m}^{-2}$; pulmonary vascular resistance 17.6 Wood units; and mixed venous oxygen saturation 58\%). Perfusion lung scanning excluded thromboembolic disease and there was no parenchymal lung disease or pleural effusions on computed tomography. His exercise capacity, as assessed by incremental shuttle walking distance (ISWD), was $180 \mathrm{~m}$ and he was in the World Health Organization (WHO) functional class (FC) III (figure 1). He was diagnosed with PAH associated with dasatinib therapy and commenced sildenafil $25 \mathrm{mg}$ three times a day.

By May 2011, he had demonstrated a good response to sildenafil with ISWD increasing to $680 \mathrm{~m}$ and his performance status had improved to WHO FC II. He subsequently commenced nilotinib $400 \mathrm{mg}$ twice a day in June 2011. In October 2011, he complained of some worsening of symptoms and sildenafil was increased to $50 \mathrm{mg}$ three times a day. His symptoms stabilised for the next 9 months, but in July 2012 he 


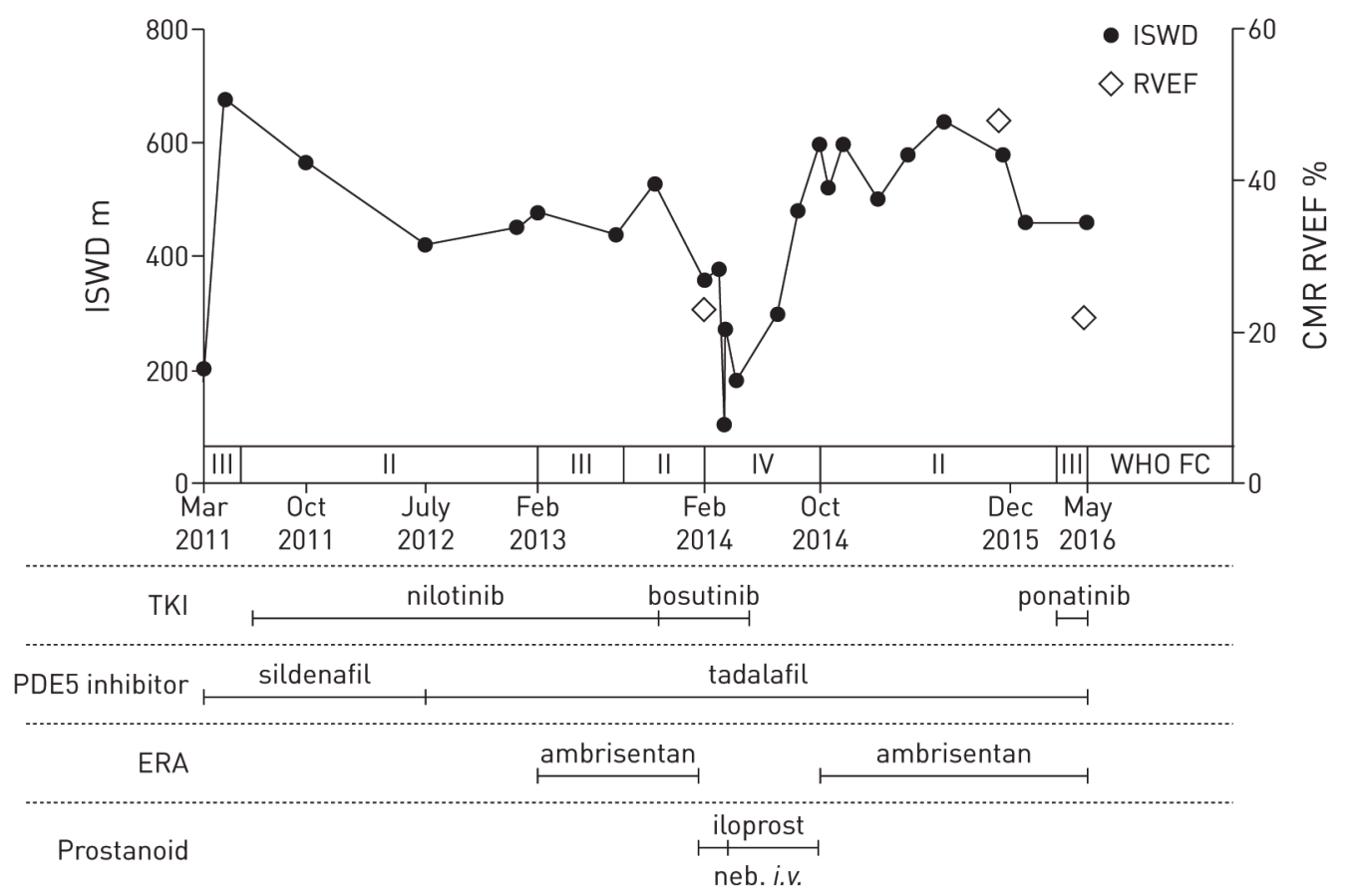

FIGURE 1 Exercise capacity, World Health Organization (WHO) functional class (FC), leukaemia therapy and pulmonary arterial hypertension therapy. ISWD: incremental shuttle walking distance; CMR: cardiac magnetic resonance; RVEF: right ventricular ejection fraction; TKI: tyrosine kinase inhibitor; PDE5: phosphodiesterase type 5; ERA: endothelin receptor antagonist; neb.: nebulised.

complained of further worsening of symptoms and sildenafil was changed to tadalafil $40 \mathrm{mg}$ once a day in case compliance was an issue. By February 2013, he had deteriorated to WHO FC III and repeat RHC demonstrated a mean pulmonary arterial pressure $57 \mathrm{mmHg}$, cardiac index $2.4 \mathrm{~L} \cdot \mathrm{min}^{-1} \cdot \mathrm{m}^{-2}$ and pulmonary vascular resistance 12.7 Wood units. Ambrisentan was added and by August 2013 he had symptomatically improved back to WHO FC II.

In December 2013, his CML treatment was switched to bosutinib $500 \mathrm{mg}$ once a day because of loss of response. By February 2014, he had clinically deteriorated to WHO FC IV and had evidence of peripheral oedema. Cardiac magnetic resonance (CMR) imaging was consistent with his WHO FC IV, demonstrating severe right ventricular (RV) dysfunction with an ejection fraction of $23 \%$. There was no evidence of pleural effusion while he had a preserved left ventricular ejection fraction of $63 \%$. Ambrisentan was switched to nebulised iloprost $(5 \mu \mathrm{g}$; seven times per day), diuretics were increased but bosutinib was continued. Because of lack of clinical improvement, he was transitioned to domiciliary intravenous iloprost in March 2014. In June 2014, he was admitted to acute care in decompensated right heart failure with peripheral oedema, a new right-sided effusion and severe dilatation of the right-sided cardiac chambers with no left atrial enlargement on imaging. He was given further diuretics and required a chest drain. Bosutinib was discontinued. His PAH therapy was unchanged.

3 months following cessation of bosutinib, he had improved markedly being in WHO FC II and by 6 months his ISWD had increased to $600 \mathrm{~m}$; i.v. iloprost was therefore discontinued and he was restarted on a combination of tadalafil and ambrisentan. CMR in December 2015 reflected his WHO FC II, demonstrating only mild RV impairment with a right ventricular ejection fraction (RVEF) of $48 \%$.

In March 2016, he was commenced on ponatinib. He was reviewed after 6 weeks of treatment because he complained of worsening breathlessness. His PAH therapy was unchanged. His ISWD was unchanged at $460 \mathrm{~m}$; however, symptomatically he was in WHO FC III. CMR was consistent with the worsening of his WHO FC, demonstrating significant deterioration compared with December 2015, with RV end-diastolic volume increasing from 157 to $220 \mathrm{~mL}$, RVEF falling to $22 \%$ and the development of more pronounced paradoxical septal motion. There was no evidence of pleural effusions. Ponatinib was stopped and he awaits further review.

Although phase 1-3 studies identified possible $\mathrm{PH}$ as an adverse reaction in four out of 870 patients treated with bosutinib [3], to our knowledge this is the first published case report linking bosutinib with PAH. Different effects of TKIs on the pulmonary vasculature in vitro, animal models and humans have been described. Imatinib was shown to have possible beneficial effects in PAH in humans although, 
because of a high rate of side-effects, most notably subdural haematomas, it has not been licensed. Although nilotinib-associated PAH has not been described, a randomised controlled trial of nilotinib in $\mathrm{PAH}$ was halted because of an increased risk of severe effects [4]. Dasatinib has been shown to reverse $\mathrm{PAH}$ in animal models; however, paradoxically dasatinib-associated $\mathrm{PAH}$ has been reported in the literature in at least 41 patients $[5,6]$. The majority of described cases of dasatinib-associated PAH have resolved or significantly regressed on cessation suggesting a vasoconstrictive pathophysiology resulting in $\mathrm{PAH}$; however, the patient in the current study had severe PAH at diagnosis despite previous cessation of dasatinib. A degree of clinical worsening was also observed while receiving nilotinib, although he responded well to the addition of a second PAH therapy despite nilotinib continuation. This worsening may have been related to persistence of dasatinib-induced vasculopathy, although an adverse effect of nilotinib cannot be discounted. Certainly, the severe deterioration we observed following commencement of bosutinib, and marked improvement on its cessation was much more clinically striking.

The kinase inhibition profile of each TKI is likely to play a key role in determining the net pulmonary vascular response. NAGARAj et al. [7] hypothesised that Src inhibition mediates the development of PAH after treatment with dasatinib or ponatinib and it is interesting to note that bosutinib, unlike imatinib and nilotinib, is also an inhibitor of Src. While Src has been implicated in the control of pulmonary vascular tone [7], in vitro data show that Src inhibition attenuates pulmonary artery smooth muscle cell proliferation and migration and reverses pulmonary arterial remodelling in both monocrotaline rat and hypoxic mouse models [8], while constitutive Src activation plays a role in endothelial dysfunction in the context of heritable PAH [9]. The paradoxical effects of TKIs observed in animal models and humans suggest that animal models are unreliable predictors of the consequence of therapeutic TKI use in patients with PAH. Further work exploring these factors and clarifying the mechanisms by which specific kinases alter pulmonary vascular cell phenotype is therefore warranted. Nevertheless, we strongly echo the recommendations by QUILOT et al. [1] that clinicians monitoring patients on TKI therapies should have a low threshold for investigating for $\mathrm{PAH}$ in patients who develop progressive breathlessness.

@ERSpublications

A patient with severe worsening of pre-existing PAH following treatment with bosutinib improved on cessation http://ow.ly/gJy7302uXL7

Peter M. Hickey ${ }^{1,2}$, Alfred A.R. Thompson ${ }^{1,2}$, Athanasios Charalampopoulos ${ }^{2}$, Charles A. Elliot ${ }^{2}$, Neil Hamilton ${ }^{2}$, David G. Kiely ${ }^{2}$, Allan Lawrie ${ }^{1}$, Ian Sabroe ${ }^{1,2}$ and Robin Condliffe ${ }^{2}$

${ }^{1}$ Dept of Infection, Immunity and Cardiovascular Disease, University of Sheffield, Sheffield, UK. ${ }^{2}$ Pulmonary Vascular Disease Unit, Sheffield Teaching Hospitals NHS Trust, Sheffield, UK.

Correspondence: Robin Condliffe, Pulmonary Vascular Disease Unit, Royal Hallamshire Hospital, Sheffield, S10 2JF, UK. E-mail: robin.condliffe@sth.nhs.uk

Received: May 192016 | Accepted after revision: July 122016 | First published online: Sept 222016

Conflict of interest: Disclosures can be found alongside this article at erj.ersjournals.com

\section{References}

1 Quilot FM, Georges M, Favrolt N, et al. Pulmonary hypertension associated with ponatinib therapy. Eur Respir J 2016; 47: 676-679.

2 Puttini M, Coluccia AM, Boschelli F, et al. In vitro and in vivo activity of SKI-606, a novel Src-Abl inhibitor, against imatinib-resistant Bcr-Abl+ neoplastic cells. Cancer Res 2006; 66: 11314-11322.

3 Electronic Medicines Compendium. Summary of product characteristics: bosulif $100 \mathrm{mg}$ and $500 \mathrm{mg}$ tablets. http://www.medicines.org.uk/emc/medicine/27795 Date last accessed: April 25, 2016. Date last updated: July 8, 2016.

4 ClinicalTrials.gov. Efficacy, safety, tolerability and pharmacokinetics (PK) of nilotinib (AMN107) in pulmonary arterial hypertension (PAH). https:/clinicaltrials.gov/ct2/show/record/NCT01179737? sect=X30156 Date last accessed: May 17, 2016. Date last updated: April 14, 2014.

5 Shah NP, Wallis N, Farber HW, et al. Clinical features of pulmonary arterial hypertension in patients receiving dasatinib. Am J Hematol 2015; 90: 1060-1064.

6 Montani D, Bergot E, Günther S, et al. Pulmonary arterial hypertension in patients treated by dasatinib. Circulation 2012; 125: 2128-2137.

7 Nagaraj C, Tang B, Bálint Z, et al. Src tyrosine kinase is crucial for potassium channel function in human pulmonary arteries. Eur Respir J 2013; 41: 85-95.

8 Pullamsetti SS, Berghausen EM, Dabral S, et al. Role of Src tyrosine kinases in experimental pulmonary hypertension. Arterioscler Thromb Vasc Biol 2012; 32: 1354-1365.

9 Prewitt AR, Ghose S, Frump AL, et al. Heterozygous null bone morphogenetic protein receptor type 2 mutations promote SRC kinase-dependent caveolar trafficking defects and endothelial dysfunction in pulmonary arterial hypertension. J Biol Chem 2015; 290: 960-971. 